Fixed Point Theory, 21(2020), No. 2, 755-766

DOI: $10.24193 /$ fpt-ro.2020.2.54

http://www.math.ubbcluj.ro/ nodeacj/sfptcj.html

\title{
SATURATED VERSIONS OF SOME FIXED POINT THEOREMS FOR GENERALIZED CONTRACTIONS
}

\author{
BURIS TONGNOI \\ Department of Mathematics, Faculty of Science \\ Naresuan University, Thailand \\ Graduate Ph.D. Program in Mathematics \\ Department of Mathematics, Faculty of Science, \\ Chiang Mai University, Chiang Mai 50200, Thailand \\ E-mail: book_buris@hotmail.com
}

\begin{abstract}
In this paper, we will give extended versions of two standard fixed point principles: one for Hardy-Rogers type operators and the other one for Ćirić type operators in complete metric space. Our results generalize similar theorems given in [9].

Key Words and Phrases: Fixed point, complete metric space, Hardy-Rogers type operators, Ćirić type operators, well-posed property, Ostrowski property, quasi-contraction.

2010 Mathematics Subject Classification: 47H10, 54H25.
\end{abstract}

Acknowledgement. This paper was written during my research stage at BabesBolyai University Cluj-Napoca. I am thankful to Professor Adrian Petrusel for his very careful guidance.

\section{REFERENCES}

[1] V. Berinde, Iterative Approximation of Fixed Points, Springer, Berlin, 2007.

[2] V. Berinde, Şt. Mă ruşter, I.A. Rus, Saturated contraction principles for non self operators, generalizations and applications, Filomat, 31(2017), 3391-3406.

[3] Lj.B. Ćirić, A generalization of Banach's contraction principle, Proc. Amer. Math. Soc., 45(1974), no. 2, 267-273.

[4] Lj.B. Ćirić, Generalized contractions and fixed-point theorems, Publ. Inst. Math., 26(1971), no. $12,19-26$.

[5] L. Janos, A converse of Banach's contraction theorem, Proc. Amer. Math. Soc., 18(1967), no. 2, 287-289.

[6] L. Janos, On contraction type mappings, Math. Balkanica, 1(1971), 52-57.

[7] I.A. Rus, Bessaga mapping, Proc. Approx. Th., Cluj-Napoca, (1984), 164-172.

[8] I.A., Rus, Generalized Contractions and Applications, Cluj Univ. Press, Cluj-Napoca, 2001.

[9] I.A. Rus, Some variants of contraction principle, generalizations and applications, Stud. Univ. Babeş-Bolyai Math., 61(2016), no. 3, 343-358.

[10] I.A. Rus, A. Petruşel, G. Petruşel, Fixed Point Theory, Cluj Univ. Press, Cluj-Napoca, 2008.

[11] T. Zamfirescu, Fixed point theorem in metric space, Arch. Math., 24(1972), 292-298.

Received: October 11, 2018; Accepted: September 2, 2019. 